\title{
Évaluation, politiques publiques, politique de recherche
}

\section{Annie Fouquet et Jacques Perriault}

\section{(2) OpenEdition}

12 Journals

Édition électronique

URL : http://journals.openedition.org/communicationorganisation/1374

DOI : 10.4000/communicationorganisation. 1374

ISSN : $1775-3546$

Éditeur

Presses universitaires de Bordeaux

\section{Édition imprimée}

Date de publication : 1 décembre 2010

Pagination : $29-40$

ISBN : 978-2-86781-743-4

ISSN : $1168-5549$

\section{Référence électronique}

Annie Fouquet et Jacques Perriault, «Évaluation, politiques publiques, politique de recherche », Communication et organisation [En ligne], 38 | 2010, mis en ligne le 01 décembre 2013, consulté le 30 avril 2019. URL : http://journals.openedition.org/communicationorganisation/1374; DOI : 10.4000/ communicationorganisation. 1374 


\title{
Évaluation, politiques publiques, politique de recherche
}

\author{
Annie fOUPU $\mathrm{T}^{1}$ et Jocques $P \in R R I A U T^{2}$
}

De nouvelles formes de participation des citoyens aux processus de décision s'imposent progressivement. Les travaux sur la «démocratie technique $»^{3}$, sur la contre-démocratie ${ }^{4}$, sur la normalisation ${ }^{5}$ révèlent la présence de plus en plus affirmée de modalités démocratiques dans le fonctionnement des groupes d'experts qui travaillent sur de multiples aspects de la vie en société. Ces modalités sont notamment la consultation des populations, la délibération, l'implication des usagers et le vote. Ces mêmes caractéristiques se retrouvent dans l'évaluation des politiques publiques, tant aux niveaux international, européen que français,: ainsi l'actuel Premier Président de la Cour des Comptes, Didier Migaud, et son prédécesseur, Philippe Seguin, de bords politiques différents, affichent pour l'institution qu'ils dirigent une même conception de l'évaluation ${ }^{6}$. Il nous apparaît donc intéressant de considérer avec cette approche la politique française de recherche et plus particulièrement son évaluation pour la situer par rapport à cette évolution générale.

Après avoir rappelé les fondamentaux généraux de l'évaluation des politiques publiques, on les appliquera au domaine de la recherche.

\section{L'évaluation de l'action publique}

Le terme d'évaluation est un « mot valise » que le sens commun charge de tous les sens possibles. Évaluer veut dire étymologiquement « apprécier la

01. Inspectrice générale des Affaires Sociales, Présidente la Société Française d'Évaluation.

02. Professeur émérite, Université Paris Ouest La Défense; Conseiller, Institut des sciences de la communication, CNRS.

03. M. Callon, P. Lascoumes, Y. Barthe, Agir sur un monde incertain. Essai sur la démocratie technique, Paris, Lavoisier, 2001.

04. Rosanvallon P., La contre-démocratie: la politique à l'âge de la défiance, Paris, Le Seuil, 2006.

05. Perriault J. «Introduction», in Perriault J., Vaguer C. (direction), Industrie de la connaissance et Internet. De la norme numérique, Paris, Éditions du CNRS, sous presse.

06. Mouterde F., Trosa S., (dir) Les nouvelles frontières de l'évaluation, Ouverture par Philippe Seguin, L'Harmattan/SFE, 2010. 
valeur ». La valeur peut être morale, esthétique, civique, économique,... On assimile souvent valeur et mesure quantitative (la bibliométrie est une de ces tentatives de mesurer la production d'un individu par un chiffre).

"Apprécier la valeur» dépend des points de vue: un même objet sera apprécié différemment selon que l'on en apprécie l'usage, la beauté, la solidité, l'origine, le prix, etc. Soit une théière chinoise Ming: elle peut être appréciée, ou bien parce qu'elle est utile pour servir du thé, ou bien parce qu'elle est ornée de figurines anciennes qu'appréciera le collectionneur; de son côté la mère de famille pourra la trouver trop petite, fragile et somme toute assez laide et de toute façon trop chère... C'est pourquoi toute évaluation doit prendre en compte la pluralité des points de vue, pour passer de la subjectivité individuelle, ou spécifique à une position sociale, à une objectivité en vue de l'intérêt général.

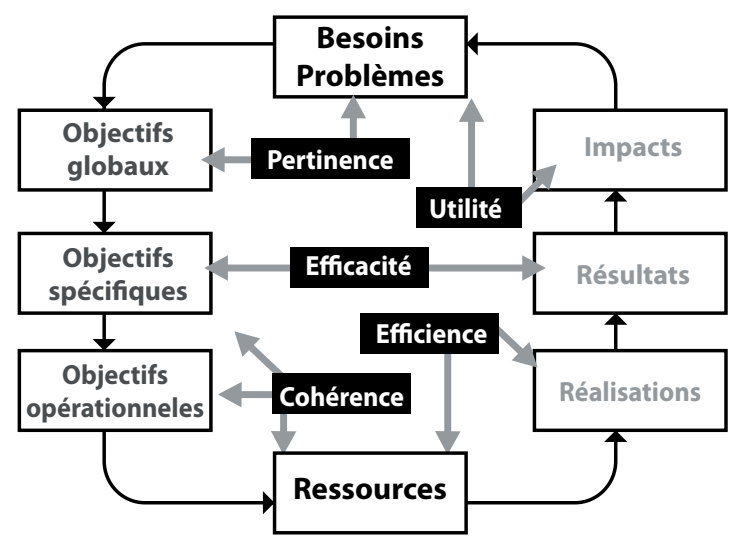

Figure 1: Le cycle de l'action publique et les registres de l'évaluation

\section{Évaluer en fonction de l'intérêt général}

Pour évaluer une action en vue de l'intérêt général, il faut que l'ensemble des parties prenantes se mettent d'accord sur le référentiel: par rapport à quoi veut-on apprécier la valeur?

L'évaluation d'une action publique (dispositif, programme ou politique) en interroge généralement la pertinence, la cohérence, l'efficacité, l'efficience, l'impact, l'attractivité (ou utilité sociale). La pertinence: l'action publique, répond-elle aux besoins qui l'ont fait naître? La cohérence interne: les moyens alloués sont-ils en rapport avec les objectifs visés? Cohérence externe: n'y a-til pas des actions menées parallèlement qui vont en sens inverse? L'efficacité: quels en ont été les résultats (à court terme, à moyen terme): les objectifs ont-ils été atteints? À quel coût (l'efficience est le ratio résultats/moyens)? 
Quel impact tout cela a-t-il eu en définitive sur la société (impacts attendus ou inattendus)? Est-ce durable?

Dans un monde parfaitement rationnel, le cycle (théorique) de l'action publique est le suivant: à un problème de société bien identifié, le décideur public met en place une politique, ensemble de programmes, déclinée en actions, avec des objectifs stratégiques, déclinés en objectifs spécifiques aux divers programmes, eux-mêmes déclinés en objectifs opérationnels. (Voir schéma).

À ces objectifs sont associés des indicateurs (qualitatifs et quantitatifs) qui permettront de savoir si l'action a été réalisée, si elle a eu des résultats, et à plus long terme des impacts. Puis d'analyser et comprendre les écarts observés avec ce qui était attendu (espéré).

\section{Les dérives du chiffre devenu nécessaire pour voir : cécité?}

Pour autant, évaluer ne se résume pas à suivre des indicateurs quantitatifs. Nos sociétés inclinent à tout vouloir mesurer par des données chiffrées, au motif que cela rend les choses comparables. Avec en plus, une focalisation sur la mesure en unités monétaires ${ }^{7}$.

Ce que montre la valorisation du travail domestique: le travail domestique est gratuit donc il n'a pas de valeur. Si l'on dit que le temps passé par les hommes et par les femmes au travail domestique est supérieur au temps passé à travailler moyennant rémunération, cela n'a pas d'impact dans le débat public. Si l'on chiffre ces temps passés en monnaie, la valorisation monétaire du travail domestique est estimée à un montant compris entre la moitié et les deux tiers du PIB. Alors, là, la presse s'en empare et découvre cette « révélation » avec stupéfaction. C'est ce qui s'est produit en 1981 lors de la publication successive par l'INSEE de deux articles présentant les résultats de l'enquête sur les Budgets temps. Le premier article en donnait les estimations en heures et minutes; il s'est fait moquer par la presse unanime à trouver dispendieux et inutile le travail de tant de « polytechniciens au mètre carré » pour trouver "ce que chacun sait ", à savoir que "à la maison, les femmes en font plus que les hommes ». Mais lorsque six mois plus tard, la même revue Économie et Statistique publie un article chiffrant ces mêmes données en francs, ce qui permet de les comparer à la valeur ajoutée produite en une année par l'ensemble des actifs, alors le sujet devient brutalement sérieux. Le travail domestique est sorti de l'invisibilité dans lequel il était resté cantonné 8 .

Pour autant la réalité est restée la même, mais le regard a changé.

07. Fouquet A., 2010, "L'usage des statistiques: de l'aide à la décision à l'évaluation des politiques publiques ». Revue française des affaires sociales, juin, numéro dirigé par Patrice Duran "L'évaluation des politiques sociales".

08. Fouquet $A$., "le travail domestique: du travail invisible au gisement d'emploi, in Laufer et alii (dir.), Masculin féminin: questions pour les sciences de l'homme, PUF, 2001. Rousse H., Roy C., "Les activités ménagères et le cycle de vie ", Économie et Statistique n 131, INSEE, avril 1981; Chadeau A., Fouquet A., « Peut-on mesurer le travail domestique? » Économie et Statistique nº 136, INSEE, septembre 1981. 


\section{$\mathrm{C} \& O \mathrm{n}^{\circ} 38$}

C'est ce que tentent de faire depuis des années, les partisans des indicateurs de richesse du PNUD qui ajoutent d'autres « valeurs » que celle de production matérielle mesurée par le PIB, telles que l'espérance de vie, la santé, l'éducation... pour comparer la qualité de vie des pays dans le monde. Façon de dire que la valeur n'est pas que monétaire.

\section{Les principes d'une évaluation menée en vue de l'intérêt général}

La confrontation des points de vue est consubstantielle à l'évaluation. Cela est vrai au niveau de l'évaluation d'une action publique qui doit être menée en vue de l'intérêt général, comme au niveau individuel dans le cadre des «entretiens d'évaluation».

Au niveau de l'intervention publique, la Société Française de l'Évaluation qui réunit tous ceux qui veulent promouvoir la culture de l'évaluation, quel que soit leur statut (commanditaires ou prestataires, acteurs ou chercheurs, publics ou privés), a adopté une Charte de l'évaluation en France (inspirée des travaux internationaux) qui pose sept principes: pluralité (des points de vue), distanciation (ne pas être juge et partie), compétence, respect des personnes, transparence, opportunité, responsabilités.

La Charte est un guide de principes généraux, largement reconnus dans le milieu professionnel. Elle invite à s'y conformer sans méconnaître les possibles contradictions entre principes dans certaines circonstances. Elle aide à prévenir les difficultés qui pourraient surgir faute d'avoir clarifié au départ les questions de principe et de déontologie.

\section{L'évaluation n'est pas du contrôle, ne se résume pas au parangonnage}

L'évaluation se distingue d'autres regards portés sur l'action publique que sont le contrôle, l'audit ou le pilotage. Contrôler l'action publique consiste à comparer l'action menée par une unité à sa conformité aux textes, lois et règlements qui en régissent les contours; la conséquence d'un contrôle est la sanction en cas de non-respect des règles. L'évaluation vise à interroger la pertinence des règles elles-mêmes et plus généralement la pertinence des objectifs: elle commence par définir le référentiel selon lequel la politique publique va être appréciée. Et la conséquence en est une amélioration de la politique menée pour mieux répondre aux besoins identifiés.

Les entretiens dits d'évaluation ont, en théorie, des points communs avec ce qui vient d'être dit sur l'évaluation de l'action publique. C'est un entretien où chacun exprime son point de vue à partir d'éléments objectifs (les réalisations, et résultats atteints, par rapport à ceux qui étaient attendus), et subjectifs (la façon dont est vécue l'action), dans un respect réciproque, en vue d'améliorer l'action. Encore faut-il que les protagonistes les mènent bien dans cet esprit.

09. Voir la Charte de l'évaluation sur le site de la Société Française de l'évaluation: www.sfe-asso.fr 
L'évaluation n'est pas du parangonnage (comparer les rangs/benchmarking). Si les indicateurs permettent de faire des palmarès, cela ne suffit pas à améliorer l'action publique. Au pays des aveugles les borgnes sont rois. L'évaluation vise à comprendre et analyser les écarts constatés entre ce qui était attendu et ce qui est observé; notamment par l'analyse des systèmes d'acteurs. Car l'action publique relève de la « chose publique » (res publica); nul n'en est l'auteur ou la victime exclusive; l'imputabilité des faits est un art difficile auquel s'attellent chercheurs et praticiens internationaux ${ }^{10}$.

\section{De l'intérêt général au bien commun : de nouvelles formes de gouvernance?}

Le processus d'évaluation requiert la prise en compte des points de vue, à divers moments: au début dans le choix du référentiel et la formulation des questions à analyser, puis une fois le constat fait et partagé, dans l'interprétation de ces résultats pour en tirer des leçons sur les améliorations à apporter. Un constat tiré d'une étude n'est pas, à lui seul, une évaluation: il n'en est qu'un élément qui demande à être intégré au système de valeurs de chacune des parties prenantes.

La confrontation des points de vue sur la chose publique est une pratique démocratique où l'intérêt général ne tombe pas de haut mais se construit avec les acteurs: elle participe de la construction du bien commun ${ }^{11}$.

\section{Évaluation de la recherche}

Le terme "évaluation de la recherche " recouvre deux réalités distinctes, même si elles entretiennent des rapports. La première réalité, la plus souvent retenue dans le monde académique, concerne l'évaluation des connaissances et de ceux qui les produisent. La seconde, qui est souvent confondue avec la précédente, a trait à la politique de recherche. Commandée et financée par l'État, c'est bien une politique publique. Qu'attendre d'une évaluation ainsi mise en œuvre?: des instantanés successifs de la politique concernée, des observations de sa performance scientifique, budgétaire, internationale, une réflexivité et une implication accrues du milieu ambiant mobilisé et des améliorations de la théorie de l'action enclenchée.

L'évaluation de la recherche est une pratique qui comporte donc deux volets: l'évaluation de la politique publique de recherche et celle de l'évaluation des connaissances et des chercheurs. Ces deux aspects sont parfois difficiles à discerner, au risque de la confusion des genres. Le ministère de la Recherche et de l'Enseignement supérieur a récemment clarifié les choses en définissant une stratégie nationale de la recherche et de l'évaluation (SNRI) avec le double affichage des priorités thématiques et du dispositif institutionnel. On

10. Voir le site de l'European Evaluation Society et son dernier Congrès à Prague, octobre 2010; ou les sites de la Commission européenne (www.evalsed.org).

11. Pierre Rosanvallon La Contre-démocratie, La politique à l'âge de la défiance, Sevil, 2006 ; Points-Essais, $n^{\circ} 598,2008$. 
en doit la préconisation à l'Agence française d'évaluation de la recherche et de l'enseignement supérieur (AERES), à partir de son expérience d'évaluation des unités de recherche et d'amélioration de la gouvernance des établissements ${ }^{12}$.

Les trois axes thématiques prioritaires sont: 1) la santé, le bien-être, l'alimentation, 2) les nanotechnologies, les urgences environnementales, les écotechnologies, 3) l'information et la communication. Les grandes fonctions du dispositif institutionnel sont également au nombre de trois:

- La fonction «Orientation », au niveau gouvernemental: élaboration et mise en œuvre de la politique nationale, définition des grandes orientations pour l'évolution du système et la répartition des ressources sur des macroobjectifs.

- La fonction « Programmation » qui traduit les macro-objectifs définis au titre de la fonction «Orientation » en priorité scientifiques et programmes de recherche, ainsi qu'en allocation de ressources pour les opérateurs ou unités de recherche. Les acteurs sont les agences de financement, les organismes de recherche et certains ministères.

- La fonction «Recherche et innovation »: fonction de production, de diffusion et de valorisation des connaissances. Elle relève des universités, des écoles, des instituts de recherche et des entreprises. ${ }^{13}$

Comme toute politique publique, la politique de recherche est composée d'éléments qui s'agrègent, de l'unité élémentaire qu'est le chercheur de base, à la politique globale en passant par l'équipe ou le laboratoire, et les programmes de recherche dans lequel ce dernier s'inscrit.

C'est un dispositif à quatre étages qui concerne respectivement la politique publique de recherche proprement dite, le processus de production des laboratoires, les connaissances produites, et enfin les chercheurs eux-mêmes. Les deux premiers étages relèvent de méthodes et de pratiques d'évaluation de l'action publique que l'on rencontre dans d'autres secteurs d'activité. Les deux derniers, par contre, relèvent de pratiques spécifiques.

\section{L'évaluation de la politique scientifique}

Lévaluation de la politique s'interroge sur la pertinence de cette politique face aux besoins de la société. Quelles sont les attentes de la société en matière de recherche? Ces attentes sont fonction de la nature du pacte entre la société et la science, pacte qui évolue au cours du temps.

$\mathrm{Au} \mathrm{XIX}^{\mathrm{e}}$ siècle, l'attente de la société à l'égard de la science s'inscrit sur la toile de fond du Progrès. Certains quotidiens, comme la Gazette de Toulouse, publient chaque semaine de larges extraits des comptes rendus de l'Académie des Sciences. Après la Seconde guerre mondiale, après Hiroshima, la dimension éthique prend une dimension importante dans les attentes de la société. Le

12. MENSR, Stratégie nationale de recherche et d'innovation, Paris, MENSR, 2009.

13. http://www.enseignementsup-recherche.gouv.fr/cid28861/ 
débat autour de Philippe Oppenheimer, le père de la bombe à hydrogène, alerte la société sur les limites que la science ne devrait pas dépasser.

Dans les années soixante-dix, émerge la problématique des rapports entre science, technique et société et son corollaire, la question du contrôle social de la technologie. Aujourd'hui, l'attente est différente, compte tenu de la mondialisation, de l'incertitude, de la démographie. On attend de la science des résultats concrets à caractère opératoire sur de grands problèmes qui concernent la santé, l'écologie, la communication et l'information notamment.

La finalité de l'attente envers la recherche semble bien être aujourd'hui un mieux-être, même s'il est problématique dans une société régie par l'incertitude ${ }^{14}$. En cela, l'activité scientifique qui aboutit à de nouvelles connaissances est régie par des politiques publiques qui pilotent ces instruments que sont les universités, le CNRS, l'INSERM, le CEA ou encore des agences, telles que l'ANR par son financement de programmes de recherche. La stratégie nationale de recherche et d'innovation, élaborée sous la tutelle du ministère de l'Enseignement supérieur et de la Recherche, a confié à ces organismes et à de nombreux chercheurs le soin d'élaborer une politique publique de recherche.

Pour évaluer cette politique de recherche, ne faudrait-il pas en considérer les objectifs tels qu'ils ressortent des définitions ministérielles puis préciser à quelle aune et de quelle façon on va les apprécier? L'application de la fonction d'évaluation décrite dans la première partie de l'article contribuerait à notre sens à améliorer l'action par le processus délibératif auquel participe chacune des parties prenantes: chercheurs, administrateurs, usagers notamment avec leurs attentes.

Ainsi mise en œuvre, l'évaluation s'inscrit dans la durée, balisée par des rencontres périodiques où se confrontent et se discutent les avis et les points de vue. Les délibérations portent non pas sur les connaissances produites mais sur la politique de recherche proprement dite, notamment sur ses objectifs, ses modalités et les valeurs sous-jacentes. Il s'agit de principes et de valeurs sociales, ce qui n'est pas du ressort des seuls experts, mais relève des forces sociales ou de la représentation nationale. Le Danemark a résolu cette question par l'instauration de conférences citoyennes, qui aident les élus à prendre position. Les points de vue cristallisés des experts peuvent être dépassés par des préconisations mesurées ou des opinions laissées jusqu'alors de côté ${ }^{15}$.

\section{L'évaluation de l'unité de recherche}

Il s'agit ici d'évaluer la qualité et la performance d'une unité de recherche ou d'un groupe de chercheurs en fonction de son mode de production du savoir.

14. Annie Fouquet «L'évaluation des politiques publiques, indispensable outil de gouvernance dans une société complexe ", in Y. Cannac et S. Trosa (dir.), La réforme dont l'État a besoin. Pour un management par la confiance et la responsabilité, Dunod, 2007.

15. François Mouterde, Controverses, évaluation, citoyens, Planète Publique, janvier 2008. 
C'est une évaluation de processus. Son référentiel comprend notamment les dimensions suivantes: l'origine du processus de recherche qui a conduit aux résultats examinés, les modalités de cette production et le mode de diffusion des résultats.

\section{L'origine des travaux}

Cette origine peut:

- s'inscrire dans une dynamique qui, à un moment donné, mobilise une communauté scientifique; aujourd'hui les réseaux sociaux, les communautés de pratiques retiennent l'attention de nombreux milieux interdisciplinaires, composés d'informaticiens, de sociologues, de cognitivistes, de chercheurs en communication.

- Répondre à des manifestations fortes de la demande sociale. Les cas actuels du réchauffement climatique et des OGM sont éclairants à ce sujet.

- S’inscrire dans la compétition ou de la coopération internationale, deux mouvements en sens contraire. Le premier s'illustre par les classements d'excellence, les Prix Nobel, le second, par la création de partenariats ou de communautés d'intérêt, de taille et d'importance variables. On peut citer à ce propos la Communauté Open Source, qui développe les logiciels libres ou encore la Communauté MPEG qui définit les standards de l'image numérique.

\section{Les modalités de la production.}

L'ambiance intellectuelle de l'unité de recherche et, plus largement du milieu, est un critère important: on retiendra notamment l'appartenance à des réseaux, la veille scientifique, la réflexivité dans le milieu (B. Jurdant), la réflexivité collaborative (J. Le Marec).

De même, le renforcement de la coopération internationale, par Internet en particulier, et l'attention portée aux polémiques, aux controverses, aux conflits de position révèlent que les chercheurs considérés sont bien au fait du nouvel état de la science, résultant de confrontations multiples (D. Wolton).

La dimension épistémologique prend aujourd'hui une importance qu'elle n'avait pas dans le passé. Une raison à cela est peut-être à rechercher dans l'accroissement considérable des travaux interdisciplinaires. Comment en effet collaborer efficacement à une tâche commune si les acteurs ne sont pas au clair sur leurs théories respectives de la connaissance et sur leurs modèles? Faute de quoi, des murailles invisibles, des « murailles de verre ", risquent de créer des espaces de réflexion étanches les uns aux autres.

Dernier critère de cette catégorie précisément, le travail interdisciplinaire souvent difficile à apprécier. Car l'interdisciplinarité elle-même a évolué. Il y a eu des stades successifs de son développement. Au stade initial, le chercheur dans une discipline recourt à des notions relevant d'une autre. C'est Claude Lévi-Strauss empruntant aux mathématiques les notions d'opérateur, de plan 
et de symétrie. Il y a eu ensuite le stade coopératif, un échange de propriétés, disait Marcel Mauss de la coopération. De nombreux programmes européens ont engendré ainsi de nombreux travaux coopératifs dont beaucoup n'ont pas abouti à de véritables productions collectives. Ce fut le cas de l'apprentissage en ligne ${ }^{16}$. Le stade actuel de l'interdisciplinarité serait celui du travail collaboratif, de la co-construction du savoir, pour employer un terme très usité aujourd'hui.

\section{Les modes de diffusion des résultats}

La question est ici d'examiner commentl'unité de recherche (ou le chercheur) communique ses résultats à la société. Cette question est particulièrement difficile aujourd'hui. De nombreux travaux ont mis en question la vulgarisation scientifique, au double motif qu'elle opère des généralisations et des mises en spectacle qui ne rendent pas compte de la réalité du travail scientifique ${ }^{17}$, d'une part, et d'autre part qu'elle est l'expression d'un refus de réflexivité, d'échange, d'intersubjectivité qui permettent l'autonomisation d'un savoir collectif ${ }^{18}$.

La multiplicité et l'hétérogénéité des destinataires de la communication scientifique accroissent la difficulté de l'exercice pour les chercheurs. Lecteurs de revues scientifiques spécialisées, visiteurs de musées scientifiques, militants d'associations (écologie, biodiversité, OGM, etc.), populations concernées par des catastrophes technologiques, élus locaux, par exemple, requièrent des déclinaisons des résultats sous des formes encore peu explorées.

Dans sa thèse, Sylvie Bresson-Gillet relate le suivi de la consultation de la population sur la mise en place du futur réacteur ITER en Provence et des difficultés pour les experts d'expliquer les propriétés et les avantages de ce type de réacteur ${ }^{19}$. Elle constate 1) que la population n'a été consultée qu'une fois la décision prise, ce qui dénote une application erronée de l'évaluation des politiques publiques; 2) que les experts présents éprouvent de grandes difficultés à répondre à certaines questions du public, qui dépassent largement un contenu strictement scientifique ou technologique.

\section{La qualité des connaissances produites}

Il ne s'agit plus ici d'évaluer une politique publique, qu'elle soit nationale, territoriale ou au sein d'une université, mais de s'intéresser de plus près à la qualité des contenus. Ici ce sont les pairs et les experts qui sont en première ligne. Les critères retenus généralement sont:

16. J. Perriault, L'accès au savoir en ligne, Paris, Éditions Odile Jacob, 2002.

17. B. Jurdant, "Écriture, réflexivité, scientificité " Sciences de la société Nº 67, 2006.

18. J. Le Marec, "Chercheur de science ", Préface à B. Jurdant, Les problèmes théoriques de la vulgarisation scientifique.

19. S. Bresson-Gille, Du débat au contrat, Communication - régulation - démocratie. Éditions l'Harmattan, sous presse. 
- la communication des résultats par les chercheurs. La qualité et la nouveauté intrinsèques des connaissances produites sont appréciées par les spécialistes du domaine, chercheurs internationaux et industriels. Une comparaison internationale des méthodes adoptées serait du plus haut intérêt;

- la poursuite de travaux sur la base des résultats examinés;

- la reconnaissance par la communauté scientifique de ce que ces résultats apportent;

- la valeur commerciale ou sociale de ces résultats, sanctionnée par des brevets ou par des applications sociales.

La tendance actuelle est de trouver des indicateurs objectifs quantitatifs en recourant notamment à la bibliométrie et à la typologie des publications hiérarchisée selon la qualité scientifique accordée aux revues. L'appréciation d'un résultat conduit ainsi à ne le retenir que s'il s'inscrit dans les formes actuellement prédominantes de la publication scientifique. Ce contre quoi s'insurgent de nombreuses communautés de chercheurs avec des opérations sur Internet de type HAL (Archives ouvertes multidisciplinaires) ou encore Creative Commons, par exemple.

Mais toutes les nouvelles connaissances ne proviennent pas de programmations préparées à l'avance. Elles surgissent souvent au gré de l'imagination et de pratiques telles que le détournement ou la sérendipité, très étudiée à l'heure actuelle, qui désigne l'art de trouver des choses que l'on ne cherche pas et de les exploiter. La photographie, la pénicilline résultent de telles pratiques, qui ne s'inscrivent, par définition, dans aucune programmation. Le classement de Shanghai et, plus généralement, les typologies des publications scientifiques accusent encore, tantôt à bon escient, tantôt non, le caractère programmatique de la production du savoir scientifique. Ying Cheng, l'un des auteurs de ce classement, vient d'annoncer que de nouveaux indicateurs pour les SHS étaient en préparation.

\section{L'évaluation des chercheurs}

Bien que ne manquant pas de défauts, le principe d'évaluation par les pairs est dominant. Le CNRS demande un compte rendu d'activité tous les deux ans, les universités tous les ans. Dans ce dernier cas, ce sont les sections du CNU qui évaluent les personnes. Dans les deux cas, un compte rendu particulier est attendu pour ceux qui ont une activité importante de gestion de la recherche. On retrouve là la distinction entre politique de recherche et production de connaissances.

\section{Conclusion}

L'évaluation des politiques publiques mobilise de plus en plus l'administration et les collectivités territoriales, tant en France qu'en Europe. Elle introduit plus de démocratie par l'association de la société civile à la 
définition même des objectifs de la politique et à son suivi. Ses instruments en sont l'objectivation des résultats et leur mise en débat et la délibération.

L'évaluation des politiques de recherche est une pratique qui a un volet d'ordre général, au niveau des politiques et de leur mise en œuvre, et un volet spécifique relatif aux connaissances et aux chercheurs. Ces deux volets ne sont pas toujours clairement distingués. Or l'un mobilise des représentants de la société, des décideurs, politiques ou administrateurs, tandis que l'autre ne concerne, en première instance, que les experts scientifiques et les chercheurs. Une réflexion s'impose sur l'application des protocoles d'évaluation aux politiques de recherche, qu'elles soient territoriales ou thématiques.

Résumé: Cet article analyse deux types d'évaluation en ce qui concerne la recherche: l'évaluation de la politique publique et celle des connaissances produites. L'évaluation d'une politique publique en interroge généralement la pertinence, la cohérence, l'efficacité, l'efficience, l'impact, l'utilité sociale. Différente du contrôle, elle prend de plus en plus un tour démocratique en y associant l'ensemble des acteurs impliqués, décideurs et usagers. En France, la politique publique de recherche prend la forme d'une stratégie nationale avec des axes thématiques prioritaires qui sert de cadre de référence à l'évaluation ainsi entendue. L'évaluation scientifique des chercheurs, des laboratoires et des connaissances qu'ils produisent est d'un tout autre ordre. Elle s'appuie sur l'appréciation par les pairs, sur des indications bibliométriques, la valeur commerciale ou sociale des résultats et sur le retentissement international.

Mots-clés : Évaluation des politiques publiques, évaluation de la production scientifique, participation démocratique, stratégie nationale de recherche, axes thématiques prioritaires, appréciation par les pairs, bibliométrie

\begin{abstract}
This article deals with two different kinds of evaluations in the field of research: the evaluation of public policies, and the evaluation of knowledge that are produced. The evaluation of a public policy is focussed on its relevancy, its consistency, its effectiveness, its performance, its impact, and its social purpose. Unlike control, it takes a rather democratic course, since it associates all the actors that are involved, whether they are key influencers or practicians. In France, public policies in the field of research tend to take the shape of a national strategy, that fixes priority thematics acting as a reference frame for evaluation. Evaluating scientific research of searchers and in laboratories, and evaluating knowlegde that are produced by searchers, mean two different things. The latter is based on the assessment by peers, on bibliometrics indicators, on the commercial and social value of the results, and on their international impact.
\end{abstract}

Keywords : Public policies evaluation, scientific production evaluation, democratic participation, national research strategy, research priority thematics, assessment by peers, bibliometrics 
